\title{
Effect of fermented milk containing Lactobacillus rhamnosus SD11 on oral microbiota of healthy volunteers: A randomized clinical trial
}

\author{
P. Rungsri, ${ }^{\star} \dagger$ N. Akkarachaneeyakorn, ${ }^{\star} \ddagger$ M. Wongsuwanlert, ${ }^{\star} †$ S. Piwat, ${ }^{\star} \ddagger$ P. Nantarakchaikul, ${ }^{\star} \ddagger$ \\ and $\mathbf{R}$. Teanpaisan* $\S^{1}$ \\ ${ }^{*}$ Common Oral Diseases and Epidemiology Research Center, \\ †Department of Conservative Dentistry, \\ fDepartment of Preventive Dentistry, and \\ §Department of Stomatology, Faculty of Dentistry, Prince of Songkla University, Hat-Yai, 90112, Thailand
}

\begin{abstract}
The aims of this study were to evaluate whether short-term consumption of fermented milk containing Lactobacillus rhamnosus SD11 affected levels of oral microbiota in vivo and whether L. rhamnosus SD11 could colonize in the human mouth. We also monitored for potential side effects of the probiotic. The applicability of using L. rhamnosus SD11 compared with Lactobacillus bulgaricus as a starter culture for fermented milk was evaluated. After informed consent, 43 healthy young adults were recruited and randomly assigned to either the probiotic or control group and received fermented milk containing $L$. rhamnosus SD11 or L. bulgaricus, respectively, once daily for $4 \mathrm{wk}$. The numbers of mutans streptococci, lactobacilli, and total bacteria in saliva were counted at baseline and then after 4 and $8 \mathrm{wk}$. An oral examination was performed at baseline and after 8 wk. The persistence of L. rhamnosus SD11 was investigated by DNA fingerprinting using arbitrary primer-PCR. Results demonstrated that statistically significant reductions in mutans streptococci and total bacteria were observed in the probiotic group compared with the control group, and the number of lactobacilli was significantly increased in both groups after receiving fermented milks. Lactobacillus rhamnosus SD11 could be detected (in $>80 \%$ of subjects) up to $4 \mathrm{wk}$ following cessation of dosing among subjects in the probiotic group. No side effects were reported. Thus, L. rhamnosus SD11 could be used as a starter culture for fermented milk. Daily consumption of $L$. rhamnosus SD11-containing fermented milk for $4 \mathrm{wk}$ may have beneficial effects on oral health by reducing salivary levels of mutans streptococci. The probiotic was apparently able to colonize the oral cavity for a
\end{abstract}

Received March 31, 2017.

Accepted June 21, 2017.

${ }^{1}$ Corresponding author: rawee.t@psu.ac.th longer time than previously reported. However, the potential benefits of probiotic L. rhamnosus SD11 on oral health require further evaluation with a larger group of volunteers in a longer-term study.

Key words: probiotics, oral health, mutans streptococci, lactobacilli

\section{INTRODUCTION}

Probiotics have been used for decades in fermented products such as fermented milk and it is accepted that probiotics can provide a health benefit, such as in the prevention and treatment of gastrointestinal and immunological disorders (Cremonini et al., 2002; Johnston et al., 2007; Lee et al., 2008). The main mechanism of action is based on enhancing the commensal microbiota and preventing colonization by true pathogens.

Some Lactobacillus strains have been previously evaluated as potential probiotics for the prevention of oral diseases such as dental caries (Cagetti et al., 2013; Laleman et al., 2014), gingivitis (Staab et al., 2009), periodontitis (Teughels et al., 2013), and halitosis (Suzuki et al., 2014). In our previous studies that screened potential probiotic strains of Lactobacillus strains derived from caries-free subjects, we found that some strains, for example, Lactobacillus paracasei SD1 and Lactobacillus rhamnosus SD11 (previously identified as Lactobacillus fermentum SD11), exhibited strong activity in inhibiting growth of oral pathogens by producing antimicrobial substances (Teanpaisan et al., 2011). Subsequently, it was found that Lactobacillus paracasei SD1 and L. rhamnosus SD11 produced bacteriocins with molecular masses of 24,028.2 and 33,000 Da, respectively (Wannun et al., 2014, 2016). Both antimicrobial proteins could inhibit growth of a wide range of oral pathogens. In an in vitro study (Piwat et al., 2015), it was shown that some strains, especially L. rhamnosus strains, could adhere well to human oral mucosa. Moreover, a reduction of cariogenic pathogens and caries risk has been demonstrated in the clinical 
trials of L. paracasei SD1 (Ritthagol et al., 2014; Teanpaisan and Piwat, 2014; Teanpaisan et al., 2015). Results confirm that a selection process to obtain a probiotic strain with good properties is needed. No randomized controlled clinical trial has yet explored the effect of L. rhamnosus SD11 on oral microbiota or its safety. Thus, the aims of this preliminary study were to evaluate whether administration of fermented milk containing L. rhamnosus SD11 affects the levels of oral microbiota in vivo and whether $L$. rhamnosus SD11 could colonize in the human mouth. We also evaluated potential side effects of the probiotic. The applicability of using L. rhamnosus SD11 as a starter culture for fermented milk was compared with use of $L$. bulgaricus.

\section{MATERIALS AND METHODS}

\section{Bacterial Strains, Culture Conditions, and Fermented Milk Preparation}

Lactobacillus rhamnosus SD11, a strain selected from caries-free subjects, was previously identified as L. fermentum SD11 using PCR-RFLP analysis of $16 \mathrm{~S}$ rRNA gene profiles and protein profiles of SDS-PAGE (Teanpaisan and Dahlen, 2006). The identity of the strain was further confirmed as L. rhamnosus SD11 by its $16 \mathrm{~S}$ rRNA gene sequences, the presence of a specific band at the same level of Lactobacillus rhamnosus GG using denaturing gradient gel electrophoresis with primers of CARP according to Piwat and Teanpaisan (2013), and lack of reaction with specific primers for $L$. fermentum strains (Dickson et al., 2005).

For fermentation, the starter culture L. rhamnosus SD11 strain or a traditional Lactobacillus delbrueckii ssp. bulgaricus (L. bulgaricus) strain (Thermophilic yogurt culture FD-DVS YC-380-Yo-Flex, Chr. Hansen, Hørsholm, Denmark) was cultured as follows. The strain was recovered from storage at $-80^{\circ} \mathrm{C}$ on de Man, Rogosa, and Sharpe (MRS) agar plates and inoculated into $50 \mathrm{~mL}$ of MRS broth overnight under anaerobic conditions $\left(80 \% \mathrm{~N}_{2}, 10 \% \mathrm{H}_{2}\right.$, and $\left.10 \% \mathrm{CO}_{2}\right)$ at $37^{\circ} \mathrm{C}$. The culture was then added to $450 \mathrm{~mL}$ of MRS broth and kept under anaerobic conditions at $37^{\circ} \mathrm{C}$ for $48 \mathrm{~h}$. Cells were harvested by centrifugation $(3,000 \times g, 5$ min) from the MRS broth and washed 3 times with $0.85 \% \mathrm{NaCl}$ before being used.

The fermented milks were prepared by the Dairy Home Industries Company, Nakornrachasrima, Thailand, and consisted of fresh milk fermented with $L$. rhamnosus SD11 or L. bulgaricus as a starter culture for the probiotic and control fermented milks, respectively. Fresh milk (1 L) was inoculated with L. rhamnosus SD11 or L. bulgaricus $\left(10^{50} \mathrm{cfu} / \mathrm{L}\right)$ and then incubated at $45^{\circ} \mathrm{C}$ for $6 \mathrm{~h}$. Fermented milks were preserved at $4^{\circ} \mathrm{C}$, and were examined for viable counts and $\mathrm{pH}$ values using a pH meter (Cyberscan pH 1000, Eutech Instruments Ltd., Singapore) every month for 6 mo.

\section{Subjects}

The study was designed as a prospective double blind, randomized, controlled trial with an experimental period of $8 \mathrm{wk}$. The protocol was approved by the Faculty of Dentistry Ethics Committee at the Prince of Songkla University, Thailand (EC5708-28-L-HR). The project was registered at http://www.clinicaltrials.in .th/ (WHO Registry Network; clinical trials identifier: TCTR20151214003; http://www.clinicaltrials.in.th/ index.php?tp $=$ regtrials\&menu $=$ trialsearch\&smenu $=$ fulltext\&task=search\&task $2=$ view $1 \& i d=1614)$.

The primary outcome was the effect of the probiotic strain on the oral microbiota. Thus, the sample size calculation was based on our previous study (Teanpaisan and Piwat, 2014), in which we first studied L. paracasei SD1. We calculated that there would be an estimated $80 \%$ power at the 0.05 level of significance using 2 -sided testing. Fifteen participants per group were needed to allow for $30 \%$ dropouts. A total sample size of at least 40 participants (20 subjects/group) was judged necessary for this study. In total, 44 subjects provided the consent forms, and 1 subject was excluded due to a history of systemic disease. Thus, the study group comprised 43 healthy nonmedicating adolescents (31 women and 12 men), 20 to $25 \mathrm{yr}$ of age (mean \pm SD: $21.86 \pm 0.83 \mathrm{yr}$ ) who volunteered after giving informed consent.

To be considered for invitation, subjects had to have caries in $\leq 2$ teeth, an absence of periodontal disease, to be nonsmoking, and have daily tooth-brushing habits using a fluoride-containing toothpaste. The exclusion criteria were (a) having habitual consumption of probiotics or xylitol, (b) having systemic antibiotic medication taken within $6 \mathrm{wk}$, (c) having an allergy to cow milk, lactose intolerance, or severe food allergy, (d) having systemic or severe chronic diseases, or (e) undergoing orthodontic treatment. All subjects underwent professional prophylaxis at the beginning of the study by a clinician.

All subjects were asked to immediately report any adverse side effects and to complete the questionnaire form after $4 \mathrm{wk}$ of fermented milk consumption.

\section{Oral Examination}

Oral examinations were performed for all subjects at baseline and at the end of the study (after $8 \mathrm{wk}$ ). The 
dental caries status (DMFT; decayed, missing, filled index) was recorded by N. A. according to WHO (1987) criteria. The plaque index (PI) and gingival index (GI) were determined by P. R. and M. W., respectively, according to Quigley and Hein (1962) and Loe and Silness (1963).

The PI evaluates the plaque revealed on the buccal and lingual nonrestored surfaces of the teeth on a scale of 0 to 5 , where $0=$ no plaque; $1=$ isolated flecks of plaque at the gingival margin; $2=$ a continuous band of plaque up to $1 \mathrm{~mm}$ at the gingival margin; 3 = plaque greater than $1 \mathrm{~mm}$ in width and covering up to one-third of the tooth surface; $4=$ plaque covering from one- to two-thirds of the tooth surface; and 5 $=$ plaque covering more than two-thirds of the tooth surface. The GI scores the marginal and interproximal tissues separately on the basis of 0 to 3 , where $0=$ normal gingiva; $1=$ mild inflammation, slight change in color and slight edema but no bleeding on probing; $2=$ moderate inflammation with redness, edema, and glazing and bleeding on probing; and $3=$ severe inflammation, marked redness and edema, and ulceration with tendency to spontaneous bleeding.

\section{Intervention}

Subjects were randomly assigned to the probiotic or control group and drank $100 \mathrm{~mL}$ of fermented milk containing probiotic L. rhamnosus SD11 or L. bulgaricus, respectively, once daily for 4 wk under observation by a clinician. The content of the drink (control or probiotic) was unknown to the subjects and to the clinician responsible for the saliva samplings. The study was blinded until the time of statistical calculations.

\section{Microbial Evaluation}

Saliva samples were taken at baseline (T0), $4 \mathrm{wk}$ (T4), and 8 wk (T8) of the study period using an oral rinse method with $10 \mathrm{~mL}$ of phosphate buffer solution (Teanpaisan and Piwat, 2014). The typical colony counts of salivary mutans streptococci and lactobacilli were evaluated using the selective media mitis salivarius bacitracin agar and MRS, respectively. Five to 10 colonies of lactobacilli on MRS plates were collected, purified, and kept at $-80^{\circ} \mathrm{C}$ for monitoring of L. rhamnosus SD11.

For total bacterial counts, salivary samples were cultured on $5 \%$ blood brain heart infusion agar for aerobic bacteria and on $5 \%$ blood brain heart infusion agar supplemented with vitamin $\mathrm{K}$ and hemin for anaerobic bacteria. All plates were incubated under appropriate conditions at $37^{\circ} \mathrm{C}$ for 24 to $48 \mathrm{~h}$.

\section{Persistence of L. rhamnosus SD11 In Vivo}

The persistence of L. rhamnosus SD11 was traced using arbitrary primer (AP)-PCR, with the enterobacterial repetitive intergenic consensus (ERIC) primers ERIC1R: forward (5'-ATGTAAGCTCCTGGGGATTCAC-3') and ERIC2: reverse (5'-AAGTAAGTGACTGG GGTGAGCG-3'). This method is useful for genotyping of Lactobacillus species and a unique DNA fingerprinting pattern is found in each individual subject (Teanpaisan and Piwat, 2014). Thus, DNA fingerprinting was analyzed to demonstrate persistence of $L$. rhamnosus SD11 among the Lactobacillus strains isolated from saliva of volunteers in both groups.

Samples of DNA of strains were prepared using a Genomic DNA Extraction Kit (RBC Bioscience, Taipei, Taiwan) following the manufacturer's protocol for gram-positive bacteria. The reaction in a $50-\mu \mathrm{L}$ mixture consisted of $100 \mathrm{ng}$ of DNA template, $1.0 \mu \mathrm{mol} / \mathrm{L}$ (each primer), $1 \times$ buffer with $2.0 \mathrm{mmol} / \mathrm{L} \mathrm{MgCl}_{2}, 1.0$ $\mathrm{U}$ of Taq polymerase, and $0.2 \mathrm{mmol} / \mathrm{L}$ of each dNTP. The mixture was subjected to 35 cycles of denaturation at $95^{\circ} \mathrm{C}$ for $1 \mathrm{~min}$, ramping to $35^{\circ} \mathrm{C}$ in $3 \mathrm{~min}$, annealing at $35^{\circ} \mathrm{C}$ for $1 \mathrm{~min}$, followed by extension at $74^{\circ} \mathrm{C}$ for 2 min, and a final extension at $74^{\circ} \mathrm{C}$ for $5 \mathrm{~min}$. The PCR products were run on a $7.5 \%$ polyacrylamide gel and stained with silver staining.

\section{Analysis of Data}

All numerical data are presented as means and standard deviations (means $\pm \mathrm{SD}$ ). The general characteristics of the volunteers between the probiotic and group at T0 (age, pH of saliva, DMFT, GI, and PI) were analyzed using the Mann-Whitney U-test, and sex was analyzed using the chi-squared test. The colony counts of mutans streptococci, lactobacilli, and total bacteria were presented as $\log _{10} \mathrm{cfu} / \mathrm{mL}$ and the comparison in the same group between $\mathrm{T} 0$ and $\mathrm{T} 8$ was analyzed using one-way ANOVA followed by the post hoc test (Bonferroni). Analysis between groups at the same time point was conducted using an independent samples $t$-test. The comparison of parameters (DMFT, GI, and PI) in the same group between T0 and T8 and between the groups at $\mathrm{T} 0$ and $\mathrm{T} 8$ were analyzed using the Wilcoxon signed-rank test and the Mann-Whitney U-test, respectively. The software package used was SPSS (SPSS Inc., Chicago, IL), and differences were considered significant $P<0.05$. 


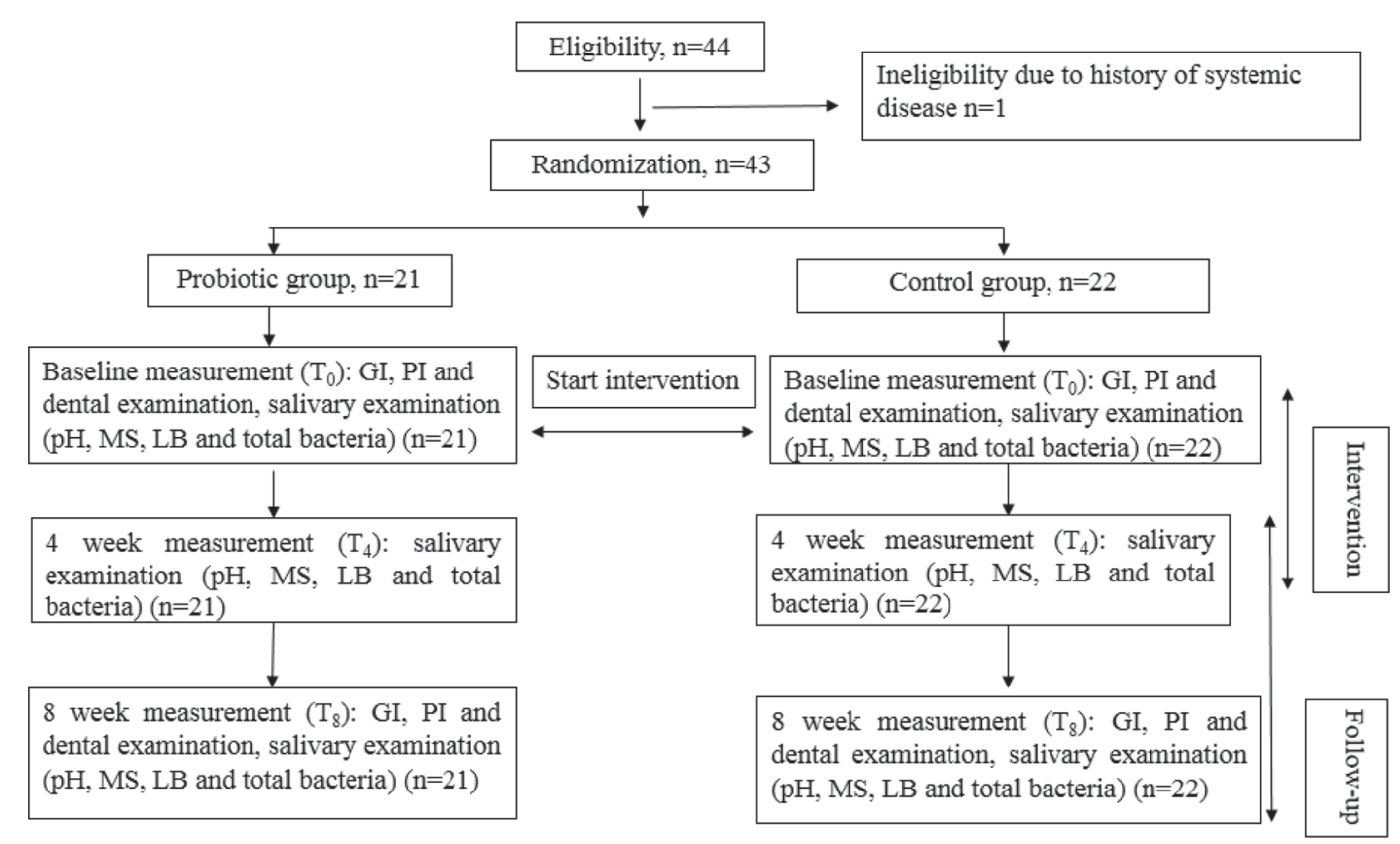

Figure 1. Flowchart of study procedures and evaluations. GI = gingival index; PI = plaque index; MS = count of mutans streptococci; LB $=$ count of lactobacilli.

\section{RESULTS}

A total 43 subjects (31 women, 12 men; 20 to $25 \mathrm{yr}$ old) fulfilled the criteria, gave informed consent, and were included in the clinical trial. No subjects dropped out during the study, and details of study activities are given in a flowchart (Figure 1). The baseline characteristics for age, sex, salivary $\mathrm{pH}$, bacterial counts, and clinical parameters between control and probiotic groups did not differ $(P>0.05)$.

The counts of mutans streptococci, lactobacilli, and total bacteria in saliva from $\mathrm{T} 0$ to $\mathrm{T} 8$ are given in Table 1. We detected no difference between the probiotic and control groups at T0. Total bacterial counts (both aerobic and anaerobic bacteria) and mutans streptococci counts were significantly $(P<0.01)$ lower in the probiotic group than in the control group at T4 and $\mathrm{T} 8$ (subjects consumed the fermented milk until T4). In the probiotic group, the mutans streptococci count was decreased $(P=0.01)$, whereas lactobacilli counts increased $(P=0.04)$ at T4 compared with T0. The number of lactobacilli in the control group also increased $(P<0.01)$ at T4 compared with T0; however, it decreased $(P=0.038)$ at $\mathrm{T} 8$ compared with T4 in the control group.

Comparison of clinical parameters of volunteers in both groups between $\mathrm{T} 0$ and $\mathrm{T} 8$ are shown in Table 2. All clinical parameters (DMFT, GI, and PI) in the

Table 1. Mean counts $\left(\log _{10} \mathrm{cfu} / \mathrm{mL}\right)$ of microbiota of volunteers in both groups at different times ${ }^{1}$

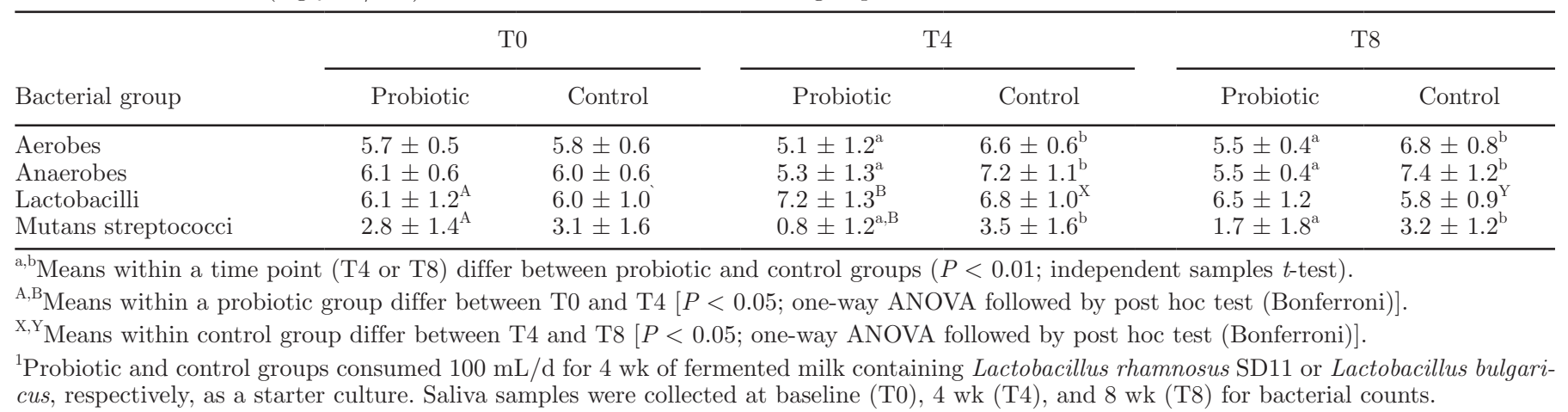


Table 2. Clinical parameters including gingival index (GI), plaque index (PI), and dental caries status (DMFT; decayed, missing, filled index) of volunteers in groups at different times ${ }^{1}$

\begin{tabular}{lccccc}
\hline & \multicolumn{2}{c}{ T0 } & & \multicolumn{2}{c}{ T8 } \\
\cline { 2 - 3 } \cline { 5 - 6 } Clinical & Probiotic & Control & & Probiotic & Control \\
parameter & $0.79 \pm 0.38$ & $0.82 \pm 0.39$ & & $0.66 \pm 0.38$ & $0.89 \pm 0.42$ \\
GI & $2.16 \pm 0.27$ & $2.14 \pm 0.20$ & & $2.07 \pm 0.45$ & $2.02 \pm 0.33$ \\
PI & $3.0 \pm 3.2$ & $3.1 \pm 3.0$ & & $3.0 \pm 3.2$ & $3.1 \pm 3.0$ \\
DMFT & & &
\end{tabular}

${ }^{1}$ Probiotic and control groups consumed $100 \mathrm{~mL} / \mathrm{d}$ for $4 \mathrm{wk}$ of fermented milk containing Lactobacillus rhamnosus SD11 or Lactobacillus bulgaricus, respectively, as a starter culture. Saliva samples were collected at baseline (T0), $4 \mathrm{wk}$ (T4), and $8 \mathrm{wk}$ (T8) for bacterial counts. Indexes are defined in the Oral Examination section.

probiotic group improved slightly after receiving fermented milk, although this change was not significant $(P>0.05)$.

Lactobacillus rhamnosus SD11 was detected in more than $80 \%$ of the subjects in the probiotic group at $\mathrm{T} 8$, and examples of the DNA fingerprint profiles of L. rhamnosus SD11 in probiotic subjects are shown in Figure 2. No side effects were reported in any group.

To examine the possibility of using L. rhamnosus SD11 as a starter culture for fermented milk, we monitored viable counts and $\mathrm{pH}$ values of fermented milk containing L. bulgaricus or L. rhamnosus SD11 for 6 mo, and the results are shown in Table 3 . We detected no difference in viable counts or $\mathrm{pH}$ values in the fermented milks over 6 mo; however, the $\mathrm{pH}$ of fermented

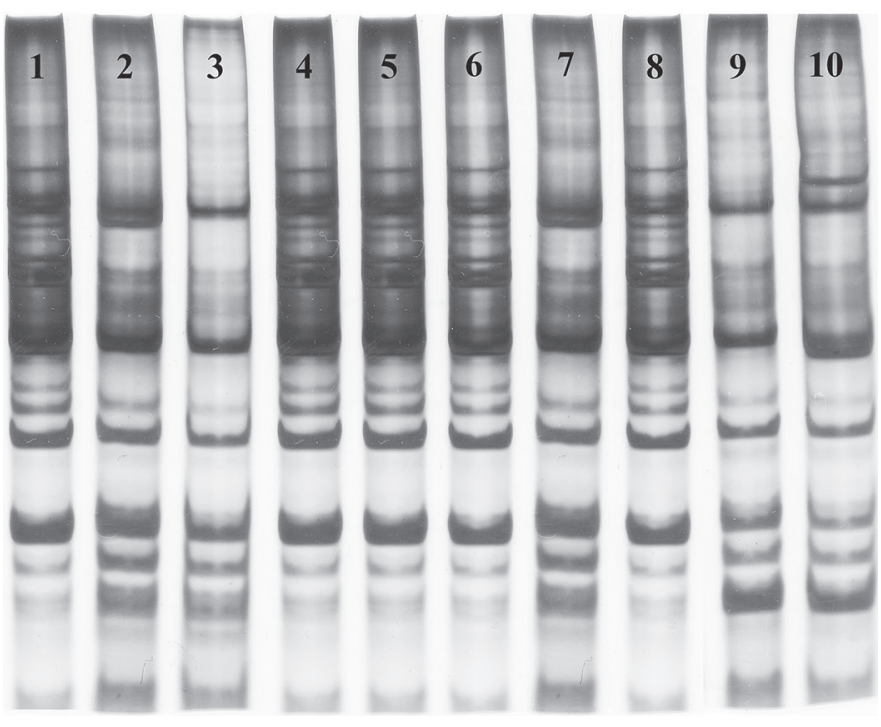

Figure 2. DNA fingerprint profiles using enterobacterial repetitive intergenic consensus (ERIC) primers of Lactobacillus rhamnosus SD11 (lane 5), and 9 Lactobacillus strains isolated from saliva of 4 subjects in the probiotic group (lanes 1 and 2, lanes 3 and 4 , lanes $6-8$, and lanes 9 and 10 belonged to 4 individual subjects, respectively). The DNA patterns in lanes $1,4,6$, and 8 were similar to that of $L$. rhamnosus SD11 in fermented milk (lane 5). milk containing $L$. bulgaricus was slightly lower than that of L. rhamnosus SD11 after 1 mo.

\section{DISCUSSION}

Recently, research on probiotics relating to oral health has received a great deal of attention. According to previous studies, probiotic interventions in the oral cavity are considered to exert their effect not only on oral pathogens but also on clinical parameters. Review articles and meta-analysis have shown that probiotic strains in different forms could reduce the number of cariogenic pathogenic mutans streptococci and reduce the risk of dental caries (Cagetti et al., 2013; Laleman et al., 2014; Gruner et al., 2016). Some studies have shown that oral administration of Lactobacillus reuteri-containing probiotic lozenges could be a useful adjunct to scaling and root planing in chronic periodontitis (Teughels et al., 2013); however, it contrasted with some others that could not find the reducing effect of probiotics on mutans streptococci (Lexner et al., 2010; Marttinen et al., 2012). It is important to recognize that the health effects of probiotics can be strain-specific, thus different results may occur from the different strains used.

This is the first study of L. rhamnosus SD11, a strain that originated from the human oral cavity, and its effect in an in vivo study. Our results indicated a significant reducing effect of the $L$. rhamnosus SD11 on both salivary mutans streptococci and total bacterial counts. This finding is in agreement with previous studies with fermented milks containing lactobacilli or bifidobacteria (Petti et al., 2001; Nikawa et al., 2004; Caglar et al., 2005; Cildir et al., 2009; Staab et al., 2009; Aminabadi et al., 2011; Ferrazzano et al., 2011). Fermented milk was chosen in this study because it has a long history of beneficial health effects in humans; for examples, in improving lactose digestion in lactoseintolerant people, affecting intestinal transit time, and stimulating the gut immune system (Elli et al., 2006). It is also a popular dairy product to deliver adequate 
Table 3. Mean counts $\left(\log _{10} \mathrm{cfu} / \mathrm{mL} ; \pm \mathrm{SD}\right)$ of Lactobacillus strains and $\mathrm{pH}$ values $( \pm \mathrm{SD})$ during 6 mo of storage of fermented milks

\begin{tabular}{|c|c|c|c|c|}
\hline \multirow[b]{2}{*}{ Time (mo) } & \multicolumn{2}{|c|}{ L. bulgaricus } & \multicolumn{2}{|c|}{ L. rhamnosus SD11 } \\
\hline & Count & $\mathrm{pH}$ & Count & $\mathrm{pH}$ \\
\hline Baseline (0) & $9.87 \pm 0.02$ & $4.60 \pm 0.14$ & $9.72 \pm 0.33$ & $4.60 \pm 0.14$ \\
\hline 1 & $9.69 \pm 0.06$ & $4.05 \pm 0.14$ & $9.51 \pm 0.18$ & $4.30 \pm 0.14$ \\
\hline 2 & $9.57 \pm 0.04$ & $4.05 \pm 0.14$ & $9.47 \pm 0.18$ & $4.30 \pm 0.14$ \\
\hline 3 & $9.25 \pm 0.07$ & $4.05 \pm 0.21$ & $9.36 \pm 0.13$ & $4.30 \pm 0.14$ \\
\hline 4 & $8.34 \pm 0.10$ & $4.05 \pm 0.21$ & $8.38 \pm 0.04$ & $4.30 \pm 0.14$ \\
\hline 5 & $8.03 \pm 0.11$ & $4.05 \pm 0.21$ & $8.01 \pm 0.18$ & $4.30 \pm 0.14$ \\
\hline 6 & $7.32 \pm 0.12$ & $4.05 \pm 0.21$ & $7.38 \pm 0.45$ & $4.30 \pm 0.14$ \\
\hline
\end{tabular}

numbers of viable probiotic cells to consumers; it is recommended that at least $100 \mathrm{~g} / \mathrm{d}$ of fermented milk containing at least $10^{6} \mathrm{cfu} / \mathrm{mL}$ be consumed regularly to gain a "probiotic effect" (Lourens-Hattingh and Viljoen, 2001). Therefore, survival of probiotics during shelf life and until consumption is an important consideration. The probiotic strain being preserved with fermented milk was supported by the present study and other studies, all of which used fermented milk as the carrier for probiotic strains, and all demonstrated that probiotics helped reduce counts of mutans streptococci (Petti et al., 2001; Nikawa et al., 2004; Caglar et al., 2005; Cildir et al., 2009; Staab et al., 2009; Aminabadi et al., 2011; Ferrazzano et al., 2011). This indicates that fermented milk containing the probiotic L. rhamnosus SD11 may be an appropriate carrier food for reduction of mutans streptococci. Survival of a probiotic strain in the host as well as in the carrier is generally considered a key feature for probiotics to preserve their healthpromoting effects.

Among previously studied Lactobacillus rhamnosus strains, L. rhamnosus GG and L. rhamnosus LC 705 were commonly strains used in clinical trials for dental caries prevention (Cagetti et al., 2013; Laleman et al., 2014; Gruner et al., 2016); both are industrial strains that have been used routinely for decades as adjunct starter cultures in dairy products. To our knowledge, these strains and traditional fermented milk starters (Lactobacillus bulgaricus) have non-oral human origins; therefore, they may not have the ability to adhere to oral epithelial cells. It has been suggested that a "probiotic must be taken in sufficient amounts on a daily basis, since probiotic strains do not permanently colonize the oral cavity or intestines" (Meurman, 2005). Aminabadi et al. (2011) showed that counts of the probiotic LGG (a strain isolated from the intestinal tract of a healthy human) were increased during the consumption period; however, counts were not maintained following cessation of consumption. This might be interpreted as a probiotic failure in bacterial competition for its colonization. The present study showed that lactobacilli increased in both groups during the intervention trial (T4), which was expected because subjects in both groups were consuming fermented milks containing lactobacilli. However, the counts of lactobacilli were slightly higher in the probiotic group than in the control group. A significant decrease in lactobacilli count was found in the control group at T8 compared with T4. This implied that L. rhamnosus SD11, originating from human oral cavity, may be better able to adhere or compete for colonization in the oral cavity than $L$. bulgaricus. Lactobacillus rhamnosus SD11 persisted in the probiotic group ( $>80 \%$ of subjects) up to $4 \mathrm{wk}$ following cessation of dosing, whereas L. rhamnosus GG could be detected in only $3.7 \%$ of subjects after $7 \mathrm{~d}$ of intervention (Yli-Knuuttila et al., 2006). These findings indicate that $L$. rhamnosus SD11 may be an appropriate probiotic strain for oral the cavity because of its ability to adhere to human oral mucosa.

The reason for the effect of probiotic L. rhamnosus SD11 on decreasing mutans streptococci and total bacteria counts is not fully known but might result from a combination of the strain's abilities to produce bacteriocin (Wannun et al., 2016) and to adhere to human oral mucosa (Piwat et al., 2015). In the current study, no significant change in clinical parameters (GI, PI, and DMFT) was found in either group. This was expected because the study was conducted over a short time and all subjects underwent professional prophylaxis at the beginning of the study. However, the short trial was sufficient to monitor the change in oral microbiota - the primary outcome. A study lasting at least $1 \mathrm{yr}$ would be required to monitor the progression of dental caries, especially in permanent teeth, and that is planned for our further study.

No side or adverse effects were reported during the trial, indicating the safety of the strain used. However, it should be noted that the sample size was limited, subjects were healthy young adults, and the study was conducted with a short intervention time. 
Although not the main purpose of this evaluation, we also examined the possibility of using $L$. rhamnosus SD11 as a starter culture for fermented milk. Results showed no significant differences in terms of viable lactobacilli counts and $\mathrm{pH}$ values for $L$. rhamnosus SD11 compared with L. bulgaricus. After 6 mo of storage, the fermented milk met the quality requirement of viable lactobacilli, having more than $10^{6} \mathrm{cfu} / \mathrm{mL}$ (Rybka and Kailasapathy, 1995) and a $\mathrm{pH}$ range of 3.7 to 4.3 (Hamann and Marth, 1983). Results indicate that $L$. rhamnosus SD11 could be used as a starter culture for fermented milk.

\section{CONCLUSIONS}

Lactobacillus rhamnosus SD11 has potential as a good probiotic strain. The daily consumption of fermented milk containing L. rhamnosus SD11 for 4 wk may have beneficial effects on oral health by reducing the salivary levels of mutans streptococci, and L. rhamnosus SD11 was apparently able to colonize the oral cavity longer than reported previously. However, the potential benefits of probiotic L. rhamnosus SD11 for oral health require further studies with a larger group of volunteers in a longer-term study.

\section{ACKNOWLEDGMENTS}

This work was supported by the National Research Council of Thailand (Bangkok; DEN580217M) and the Prince of Songkla University research fund, Super clusters (Hat Yai, Thailand; DEN600729S).

\section{REFERENCES}

Aminabadi, N. A., L. Erfanparast, A. Ebrahimi, and S. G. Oskouei 2011. Effect of chlorhexidine pretreatment on the stability of salivary lactobacilli probiotic in six- to twelve-year-old children: A randomized controlled trial. Caries Res. 45:148-154.

Cagetti, M. G., S. Mastroberardino, E. Milia, F. Cocco, P. Lingström, and G. Campus. 2013. The use of probiotic strains in caries prevention: A systematic review. Nutrients 5:2530-2550.

Caglar, E., N. Sandalli, S. Twetman, S. Kavaloglu, S. Ergeneli, and S. Selvi. 2005. Effect of yogurt with Bifidobacterium DN-173010 on salivary mutans streptococci and lactobacilli in young adults. Acta Odontol. Scand. 63:317-320.

Cildir, S. K., D. Germec, N. Sandalli, F. I. Ozdemir, T. Arun, S. Twetman, and E. Caglar. 2009. Reduction of salivary mutans streptococci in orthodontic patients during daily consumption of yoghurt containing probiotic bacteria. Eur. J. Orthod. 31:407-411.

Cremonini, F., S. Di Caro, E. C. Nista, F. Bartolozzi, G. Capelli, G. Gasbarrini, and A. Gasbarrini. 2002. Meta-analysis: The effect of probiotic administration on antibiotic-associated diarrhoea. Aliment. Pharmacol. Ther. 16:1461-1467.

Dickson, E. M., M. P. Riggio, and L. Macpherson. 2005. A novel species-specific PCR assay for identifying Lactobacillus fermentum. J. Med. Microbiol. 54:299-303.

Elli, M., M. L. Callegari, S. Ferrari, E. Bessi, D. Cattivelli, S. Soldi, L. Morelli, N. G. Feuillerat, and J. M. Antoine. 2006. Survival of yogurt bacteria in the human gut. Appl. Environ. Microbiol. 72:5113-5117.

Ferrazzano, G. F., T. Cantile, G. Sangianantoni, I. Amato, and A. Ingenito. 2011. The effects of short-term consumption of commercial yogurt on salivary mutans streptococci and lactobacilli counts: An in vivo investigation. Eur. J. Clin. Nutr. 65:1170-1172.

Gruner, D., S. Paris, and F. Schwendicke. 2016. Probiotics for managing caries and periodontitis: Systematic review and meta-analysis. J. Dent. 48:16-25.

Hamann, W. T., and E. H. Marth. 1983. Survival of Streptococcus thermophilus and Lactobacillus bulgaricus in commercial and experimental yogurts. J. Food Prot. 47:781-786.

Johnston, B. C., A. L. Supina, M. Ospina, and S. Vohra. 2007. Probiotics for the prevention of pediatric antibiotic-associated diarrhea. Cochrane Database Syst. Rev. 18:CD004827.

Laleman, I., V. Detailleur, D. E. Slot, V. Slomka, M. Quirynen, and W. Teughels. 2014. Probiotics reduce mutans streptococci counts in humans: A systematic review and meta-analysis. Clin. Oral Investig. 18:1539-1552.

Lee, J., D. Seto, and L. Bielory. 2008. Meta-analysis of clinical trials of probiotics for prevention and treatment of pediatric atopic dermatitis. J. Allergy Clin. Immunol. 121:116-121.e11.

Lexner, M. O., S. Blomqvist, G. Dahlén, and S. Twetman. 2010. Microbiological profiles in saliva and supragingival plaque from caries-active adolescents before and after a short-term daily intake of milk supplemented with probiotic bacteria-A pilot study. Oral Health Prev. Dent. 8:383-388.

Loe, H., and J. Silness. 1963. Periodontal disease in pregnancy. I. Prevalence and severity. Acta Odontol. Scand. 21:533-551.

Lourens-Hattingh, A., and B. C. Viljoen. 2001. Yogurt as probiotic carrier food. Int. Dairy J. 11:1-17.

Marttinen, A., A. Haukioja, S. Karjalainen, L. Nylund, R. Satokari, C. Öhman, P. Holgerson, S. Twetman, and E. Söderling. 2012 Short-term consumption of probiotic lactobacilli has no effect on acid production of supragingival plaque. Clin. Oral Investig. 16:797-803.

Meurman, J. H. 2005. Probiotics: Do they have a role in oral medicine and dentistry? Eur. J. Oral Sci. 113:188-196.

Nikawa, H., S. Makihira, H. Fukushima, H. Nishimura, Y. Ozaki, K. Ishida, S. Darmawan, T. Hamada, K. Hara, A. Matsumoto, T. Takemoto, and R. Aimi. 2004. Lactobacillus reuteri in bovine milk fermented decreases the oral carriage of mutans streptococci. Int J. Food Microbiol. 95:219-223.

Petti, S., G. Tarsitani, and A. S. D'Arca. 2001. A randomized clinical trial of the effect of yoghurt on the human salivary microflora. Arch. Oral Biol. 46:705-712.

Piwat, S., B. Sophatha, and R. Teanpaisan. 2015. An assessment of adhesion, aggregation, and surface charges of Lactobacillus strains derived from the human oral cavity. Lett. Appl. Microbiol. 61:98105.

Piwat, S., and R. Teanpaisan. 2013. 16S rRNA PCR-denaturing gradient gel electrophoresis of oral Lactobacillus casei group and their phenotypic appearances. ISRN Microbiol. 2013:342082. https:// doi.org / 10.1155/2013/342082.

Quigley, G. A., and J. W. Hein. 1962. Comparative cleansing efficiency of manual and power brushing. J. Am. Dent. Assoc. 65:26-29.

Ritthagol, W., C. Saetang, and R. Teanpaisan. 2014. Effect of probiotics containing Lactobacillus paracasei SD1 on salivary mutans streptococci and lactobacilli in orthodontic cleft patients: A double-blinded, randomized, placebo-controlled study. Cleft Palate Cranio. J. 51:257-263.

Rybka, S., and K. Kailasapathy. 1995. The survival of culture bacteria in fresh and freeze-dried AB yogurts. Aust. J. Dairy Technol. 50:58-60.

Staab, B., S. Eick, G. Knöfler, and H. Jentsch. 2009. The influence of a probiotic milk drink on the development of gingivitis: A pilot study. J. Clin. Periodontol. 36:850-856.

Suzuki, N., M. Yoneda, K. Tanabe, A. Fujimoto, K. Iha, K. Seno, K. Yamada, T. Iwamoto, Y. Masuo, and T. Hirofuji. 2014. Lactobacillus salivarius WB21-containing tablets for the treatment of oral 
malodor: A double-blind, randomized, placebo-controlled crossover trial. Oral Surg. Oral Med. Oral Pathol. Oral Radiol. 117:462-470.

Teanpaisan, R., and G. Dahlen. 2006. Use of polymerase chain reaction techniques and sodium dodecyl sulfate-polyacrylamide gel electrophoresis for differentiation of oral Lactobacillus species. Oral Microbiol. Immunol. 21:79-83.

Teanpaisan, R., and S. Piwat. 2014. Lactobacillus paracasei SD1, a novel probiotic, reduces mutans streptococci in human volunteers: A randomized placebo-controlled trial. Clin. Oral Investig. 18:857862 .

Teanpaisan, R., S. Piwat, and G. Dahlén. 2011. Inhibitory effect of oral Lactobacillus against oral pathogens. Lett. Appl. Microbiol. $53: 452-459$.

Teanpaisan, R., S. Piwat, S. Tianviwat, B. Sophatha, and T. Kampoo. 2015. Effect of long-term consumption of Lactobacillus paracasei SD1 on reducing mutans streptococci and caries risk: A randomized placebo-controlled trial. Dent. J. 3:43-54.
Teughels, W., A. Durukan, O. Ozcelik, M. Pauwels, M. Quirynen, and M. C. Haytac. 2013. Clinical and microbiological effects of Lactobacillus reuteri probiotics in the treatment of chronic periodontitis: A randomized placebo-controlled study. J. Clin. Periodontol. 40:1025-1035.

Wannun, P., S. Piwat, and R. Teanpaisan. 2014. Purification and characterization of bacteriocin produced by oral Lactobacillus paracasei SD1. Anaerobe 27:17-21.

Wannun, P., S. Piwat, and R. Teanpaisan. 2016. Purification, characterization, and optimum conditions of fermencin SD11, a bacteriocin produced by human orally Lactobacillus fermentum SD11. Appl. Biochem. Biotechnol. 179:572-582.

WHO. 1987. Oral health surveys: Basic methods. World Health Organization, Geneva, Switzerland.

Yli-Knuuttila, H., J. Snäll, K. Kari, and J. H. Meurman. 2006. Colonization of Lactobacillus rhamnosus GG in the oral cavity. Oral Microbiol. Immunol. 21:129-131. 